\title{
Best $N$ Term Approximation Spaces for Tensor Product Wavelet Bases
}

\author{
Pál-Andrej Nitsche
}

\begin{abstract}
We consider best $N$ term approximation using anisotropic tensor product wavelet bases ("sparse grids"). We introduce a tensor product structure $\otimes_{q}$ on certain quasi-Banach spaces. We prove that the approximation spaces $A_{q}^{\alpha}\left(L_{2}\right)$ and $A_{q}^{\alpha}\left(H^{1}\right)$ equal tensor products of Besov spaces $B_{q}^{\alpha}\left(L_{q}\right)$, e.g.,

$$
A_{q}^{\alpha}\left(L_{2}\left([0,1]^{d}\right)\right)=B_{q}^{\alpha}\left(L_{q}([0,1])\right) \otimes_{q} \cdots \otimes_{q} B_{q}^{\alpha}\left(L_{q}([0,1])\right) .
$$

Solutions to elliptic partial differential equations on polygonal/polyhedral domains belong to these new scales of Besov spaces.
\end{abstract}

\section{Introduction}

In this paper, we investigate approximation spaces for best $N$ term approximation using tensor product bases of one-dimensional hierarchical wavelet bases. These tensor product bases are anisotropic, i.e., high frequencies in some directions can be tensorized with low frequencies in other directions. Subsets of these bases span the so-called sparse grid spaces, which have been successfully applied to the numerical treatment of elliptic partial differential equations (PDEs), to data mining, or to high-dimensional integration.

Given a basis $\left\{\varphi_{k}: k \in \mathbf{N}\right\}$ of a function space $V$ with norm $\|\cdot\|_{V}$, a best $N$ term approximation $g_{N}$ to a function $f \in V$ realizes the following infimum:

$$
\inf \left\{\|f-g\|_{V}: g=\sum_{i=1}^{N} c_{i} \varphi_{k_{i}}\right\} .
$$

The space in which the approximation is sought is the nonlinear manifold consisting of all linear combinations of the given basis with at most $N$ terms; for this reason best $N$ term approximation is often called nonlinear approximation.

Best $N$ term approximation is an important theoretical tool in the mathematical treatment of adaptive numerical approximation, because it gives upper bounds: if the sequence $\left(g_{N}\right)$ of best $N$ term approximations converges at a certain rate $\alpha$, no adaptive scheme (using this particular basis) can do better. On the other hand, one should strive to

Date received: September 28, 2004. Date revised: July 8, 2005. Date accepted: July 11, 2005. Communicated by Wolfgang Dahmen. Online publication: March 15, 2006.

AMS classification: 41A25, 41A63.

Key words and phrases: Best $N$ term approximation, Tensor product approximation, Sparse grids, Besov spaces. 
construct adaptive approximation schemes which reproduce the rates achieved by best $N$ term approximation.

Best $N$ term approximation has been successfully employed in the mathematical analysis of adaptive wavelet methods for elliptic PDEs, see, e.g., [6] and also papers [7], [8], and [3]. In [6], the authors construct an adaptive scheme, for isotropically supported multidimensional wavelet bases, which produces an approximation to the solution to an elliptic PDE at the asymptotic rate of best $N$ term approximation using this particular basis. For instance, the algorithm given in [6] yields an approximation rate in the $H^{1}$ norm of $\frac{1}{2}$ for solutions to smooth uniformly elliptic PDEs on two-dimensional polygonal domains using isotropically supported piecewise bilinear ansatz functions; the rate $\frac{1}{2}$ is optimal with respect to this isotropically supported wavelet basis.

However, it has been numerically observed (e.g., in the group of Zenger, see [1]), that adaptive approximation using sparse grid spaces can approximate certain classes of singularities arising in elliptic PDEs due to corners, edges, etc., of polygonal and polyhedral domains at substantially higher rates. For the elliptic PDE from above on a two-dimensional polygonal domain, this yields approximation rates in the $H^{1}$-norm of $1-\varepsilon$ for arbitrarily small $\varepsilon>0$, using piecewise bilinear ansatz functions as well.

This has been rigorously proven in an a priori wavelet context in [20]: solutions to elliptic PDEs (with smooth data) in polyhedral domains (in dimensions 2 and 3 ) can be approximated by sparse grid wavelet spaces (appropriately refined toward the singular support) built from biorthogonal spline wavelets of local polynomial degree $p$ at any rate $<p+1$ with respect to the $L_{2}$-norm and at any rate $<p$ with respect to the $H^{1}$-norm, thus overcoming the so-called curse of dimension.

The higher the dimension of the underlying domain of the elliptic PDE the more is possibly to gain using tensor product bases instead of isotropically supported bases. Accompanying the rise in computing power, there has been an increased interest in the numerical treatment of high-dimensional elliptic PDEs in recent years. High-dimensional elliptic PDEs arise, e.g., in mathematical homogenization, molecular physics, quantum chemistry, and mathematical finance, to name but a few, and they often come in some kind of product of tensor product structure.

The purpose of the present paper is to provide regularity notions relevant for the application of adaptive numerical strategies to the approximation of solutions to (highdimensional) elliptic PDEs using tensor product ("sparse grid") bases.

We characterize the class of functions which can be approximated in $L_{2}$ or $H^{1}$ by anisotropic tensor product bases ("sparse grid bases") at a rate $\alpha$. We prove that this class is a tensor product of appropriate one-dimensional Besov spaces. The spaces in question cease to be Banach spaces but are quasi-Banach spaces satisfying only a generalized triangle inequality. A large part of this paper deals with the construction of a tensor product structure on this type of quasi-Banach space, which is new and interesting in its own right.

Using [20], we obtain Besov regularity results for solutions to elliptic PDEs in terms of these new "anisotropic" Besov spaces, corresponding to approximation rates using nonlinear (adaptive) approximation in a tensor product setting. Contrary to the isotropic case, where the presence of anisotropic singularities in dimension $d \geq 3$ leads to restrictions on the possible order of isotropic Besov smoothness, in the tensor product setting the order of Besov smoothness is unlimited irrespective of the dimension. For a more detailed discussion, see Remark 8. 
The adaptive realization of best $N$ term approximation in the context of elliptic operator equations, as developed in [6], [7], [8], [3], applies to the tensor product setting as well when dealing with elliptic operators of tensor product form, see [21]. This means that for these problems any order of approximation can be recovered from the unlimited order of anisotropic Besov smoothness with the aid of these adaptive schemes. This offers already in dimension $d \geq 3$ an advantage over established isotropic approaches.

For the sparse grid spaces built from the Haar system, Oswald has already considered best $N$ term approximation as well as approximability of certain singularity functions, see [22].

\section{Notions from Approximation Theory}

In this section, we introduce the notation used throughout this paper. An excellent survey on linear and nonlinear approximation theory is [15]; see also [26].

Let $H$ be a separable Hilbert space with norm $\|\cdot\|_{H}$, and let $\left\{\psi_{k}: k \in I\right\} \subset H$ for some index set $I$ be a basis of $H$, i.e., the completion of the linear span of $\left\{\psi_{k}: k \in I\right\}$ (with respect to $\|\cdot\|_{H}$ ) equals $H$ :

$$
\overline{\operatorname{span}\left\{\psi_{k}: k \in I\right\}}=H .
$$

We denote the nonlinear manifolds, from which approximation takes place, by

$$
\Sigma_{N}:=\left\{\sum_{k \in \Lambda} c_{k} \psi_{k}: \Lambda \subset I, \# \Lambda \leq N\right\} .
$$

The (nonlinear) space $\Sigma_{N}$ consists of all linear combinations of functions from the set $\left\{\psi_{k}: k \in I\right\}$ with at most $N$ terms.

For a function $f \in H$, the approximation error $\sigma_{N}(f)_{H}$ is defined by

$$
\sigma_{N}(f)_{H}:=\inf _{S \in \Sigma_{N}}\|f-S\|_{H} .
$$

For real $\alpha>0$ and $0<q \leq \infty$, the approximation space $A_{q}^{\alpha}(H)$ is defined by

$$
A_{q}^{\alpha}(H):=\left\{f \in H:|f|_{A_{q}^{\alpha}(H)}<\infty\right\},
$$

where

$$
|f|_{A_{q}^{\alpha}(H)}:=\left(\sum_{N \in \mathbf{N}}\left(N^{\alpha} \sigma_{N}(f)_{H}\right)^{q} \frac{1}{N}\right)^{1 / q}
$$

for $0<q<\infty$, and

$$
|f|_{A_{\infty}^{\alpha}(H)}:=\sup _{N \geq 1} N^{\alpha} \sigma_{N}(f)_{H}
$$

for $q=\infty$. We set $\|f\|_{A_{q}^{\alpha}(H)}=|f|_{A_{q}^{\alpha}(H)}+\|f\|_{H}$.

There hold the inclusions

$$
A_{q}^{\alpha}(H) \subset A_{p}^{\alpha}(H), \quad 0<q<p \leq \infty .
$$


All the spaces $A_{q}^{\alpha}(H)$ correspond roughly to an asymptotic decrease of the error of the best $N$ term approximation (at least) like $\mathcal{O}\left(N^{-\alpha}\right)$. Note that the approximation spaces $A_{q}^{\alpha}(H)$ depend implicitly (but decisively) on the chosen basis $\left\{\psi_{k}: k \in I\right\}$.

One of the basic tasks in approximation theory is to characterize the approximation spaces $A_{q}^{\alpha}(H)$, ideally by classical spaces like $C^{k}$, Sobolev, or Besov spaces. One possibility is to prove the so-called Jackson and Bernstein inequalities for some appropriate second space $X$ :

The Jackson inequality: $\sigma_{N}(f)_{H} \leq C N^{-r}\|f\|_{X}$ for all $f \in X$ and $N \in \mathbf{N}$.

The Bernstein inequality: $\|S\|_{X} \leq C N^{r}\|S\|_{H}$ for all $S \in \Sigma_{N}$ and $N \in \mathbf{N}$.

Then one can characterize the approximation spaces as interpolation spaces (which are usually better understood), see Theorem 1 in [15].

Theorem 1. If the Jackson and Bernstein inequalities are valid for some appropriate space $X$ then, for each $0<\alpha<r$ and $0<q<\infty$, the following relation holds between approximation and interpolation spaces:

$$
A_{q}^{\alpha}(H)=(H, X)_{\alpha / r, q}
$$

(equivalent norms).

Here $(H, X)_{\theta, q}$ are the so-called real interpolation spaces, which consist of all functions $f$ for which

$$
\left(\int_{0}^{\infty}\left(t^{-\theta} K(f, t)\right)^{q} \frac{d t}{t}\right)^{1 / q}
$$

is finite, where

$$
K(f, t)=\inf _{g \in X}\|f-g\|_{H}+t|g|_{X}
$$

is the $K$ functional. For more details on interpolation spaces, see, e.g., [4], [24].

In the following we will encounter Besov spaces which play an important rôle in best $N$ term approximation. We briefly recall their definition and basic relations. Let $\alpha>0$ and $0<p, q \leq \infty$. Let $r$ be the smallest integer larger than $\alpha$. Then a function $f$ is in the Besov space $B_{q}^{\alpha}\left(L_{p}(\Omega)\right)$ if

$$
|f|_{B_{q}^{\alpha}\left(L_{p}(\Omega)\right)}:=\left(\int_{0}^{\infty}\left(t^{-\alpha} \omega_{r}(f, t)_{p}\right)^{q} \frac{d t}{t}\right)^{1 / q}<\infty
$$

for $0<q<\infty$, and for $q=\infty$ if

$$
|f|_{B_{\infty}^{\alpha}\left(L_{p}(\Omega)\right)}:=\sup _{t>0} t^{-\alpha} \omega_{r}(f, t)_{p}<\infty
$$

We set $\|f\|_{B_{q}^{\alpha}\left(L_{p}(\Omega)\right)}:=|f|_{B_{q}^{\alpha}\left(L_{p}(\Omega)\right)}+\|f\|_{L_{p}(\Omega)}$. Here $\omega_{r}(f, t)_{p}=\sup _{|h| \leq t}\left\|\Delta_{h}^{r} f\right\|_{L_{p}(\Omega)}$ is the $r$ th-order modulus of continuity and $\Delta_{h}^{r}$ is the $r$ th power of the difference operator $\Delta_{h} f(x)=f(x+h)-f(x)$, where we set $\Delta_{h}^{r} f(x):=0$ when $x+s h \notin \Omega$ for some $s \in[0, r]$. 
We have

$$
\left(L_{p}(\Omega), W^{r}\left(L_{p}(\Omega)\right)\right)_{\theta, q}=B_{q}^{\theta r}\left(L_{p}(\Omega)\right), \quad 0<\theta<1, \quad 0<q \leq \infty,
$$

where $W^{r}\left(L_{p}(\Omega)\right)$ is the Sobolev space of order $r$ built on $L_{p}(\Omega)$.

We will almost exclusively be interested in the scale of Besov spaces $B_{q}^{\alpha}\left(L_{q}(\Omega)\right)$ with $q^{-1}=\alpha+\frac{1}{2}$. Interpolation on this scale yields again a space from this scale:

$$
\left(L_{2}(\Omega), B_{q}^{\alpha}\left(L_{q}(\Omega)\right)_{\theta, s}=B_{s}^{\theta \alpha}\left(L_{s}(\Omega)\right) \quad \text { if } \quad s^{-1}=\theta \alpha+\frac{1}{2} .\right.
$$

We will need a further result on best $N$ term approximation in $\ell_{2}\left(\mathbf{N}^{k}\right)$ (Theorem 4 in [15]).

Theorem 2. For best $N$ term approximation in $\ell_{2}\left(\mathbf{N}^{k}\right)$, a vector $c$ is in $A_{q}^{\alpha}\left(\ell_{2}\left(\mathbf{N}^{k}\right)\right)$ if and only if $c$ is in the Lorentz sequence space $\ell_{\tau, q}$ with $\tau^{-1}=\alpha+\frac{1}{2}$.

For convenience, we give the definition and some properties of Lorentz sequence spaces in the following remark (see [14]).

Remark 1. Let $0<p<\infty$ and $0<q \leq \infty$. For a sequence $x=\left(x_{n}\right)$ define

$$
\|x\|_{\ell_{p, q}(\mathbf{N})}:= \begin{cases}\left(\sum_{n=1}^{\infty}\left(x_{n}^{*}\right)^{q} n^{q / p-1}\right)^{1 / q} & \text { for } q<\infty, \\ \sup _{n}\left(x_{n}^{*} n^{1 / p}\right) & \text { for } q=\infty,\end{cases}
$$

where $x^{*}=\left(x_{n}^{*}\right)$ is the decreasing rearrangement of $x$.

Then the Lorentz sequence space $\ell_{p, q}(\mathbf{N})$ is

$$
\ell_{p, q}(\mathbf{N})=\left\{x:\|x\|_{\ell_{p, q}(\mathbf{N})}<\infty\right\} .
$$

If $p=q$, then $\ell_{p, q}(\mathbf{N})=\ell_{p, p}(\mathbf{N})=\ell_{p}(\mathbf{N})$.

If $q_{1}<q_{2}$, then $\ell_{p, q_{1}}(\mathbf{N}) \subset \ell_{p, q_{2}}(\mathbf{N})$.

If $p_{1}<p_{2}$, then $\ell_{p_{1}, q}(\mathbf{N}) \subset \ell_{p_{2}}(\mathbf{N})$ for all $q \in(0, \infty]$.

The Lorentz sequence spaces arise as interpolation spaces of $\ell_{p}(\mathbf{N})$ spaces:

$$
\begin{aligned}
\left(\ell_{r}(\mathbf{N}), \ell_{s}(\mathbf{N})\right)_{\theta, q} & =\ell_{p, q}(\mathbf{N}) \\
\text { for } p^{-1} & =(1-\theta) r^{-1}+\theta s^{-1} \text { and } \quad 0<q \leq \infty, \quad 1 \leq r, s .
\end{aligned}
$$

The space $\ell_{p, \infty}(\mathbf{N})$ is also called weak- $\ell_{p}$. A sequence in $\ell_{p, \infty}(\mathbf{N})$ is characterized by

$$
x_{n}^{*} \lesssim n^{-1 / p} \text {. }
$$

\section{Tensor Product Wavelet Basis}

In this section we describe a class of bases for which our best $N$ term approximation result will hold. These bases are tensor products of one-dimensional wavelet bases; the 
linear span of certain subsets of these basis functions is called a "sparse grid." The domain $\Omega$ under consideration will be the unit cube $[0,1]^{d}$.

Let $\left\{\psi_{j k}: j \in \mathbf{N}, k=1, \ldots, K_{j}\right\}, K_{j} \lesssim 2^{j}$, be a basis for $L_{2}([0,1])$ such that the following norm equivalences hold:

$$
\begin{gathered}
\left\|\sum_{j, k} c_{j k} \psi_{j k}\right\|_{B_{q}^{\alpha}\left(L_{q}([0,1])\right)} \sim\left\|\left(2^{j(\alpha-1 / q+1 / 2)} c_{j k}\right)\right\|_{\ell_{q}(\mathbf{N})}, \\
0<\alpha<\alpha_{0}, \quad q \text { in an open interval around }\left(\alpha+\frac{1}{2}\right)^{-1},
\end{gathered}
$$

and

$$
\left\|\sum_{j, k} c_{j k} \psi_{j k}\right\|_{L_{2}([0,1])} \sim\left\|\left(c_{j k}\right)\right\|_{\ell_{2}(\mathbf{N})} .
$$

For simplicity we will call such a basis an $\alpha_{0}$-basis.

Any wavelet system for which:

(i) $\psi_{j k} \in B_{q}^{\beta}\left(L_{q}([0,1])\right)$ for some $\beta>\alpha$;

(ii) $\psi_{j k}$ has $r$ vanishing moments with $r>\alpha$; and

(iii) $r>1 / q-1$ if $q<1$;

satisfies the norm equivalence

$$
\left\|\sum_{j, k} c_{j k} \psi_{j k}\right\|_{B_{q}^{\alpha}\left(L_{q}([0,1])\right)} \sim\left\|\left(2^{j(\alpha-1 / q+1 / 2)} c_{j k}\right)\right\|_{\ell_{q}(\mathbf{N})} ;
$$

see, e.g., [15] or [9].

So, for instance, the wavelet systems constructed in [12] or [13], which are biorthogonal spline wavelets of local degree $p$ with $p+1$ vanishing moments, are $\alpha_{0}$-bases for $\alpha_{0}=p+1$. The following illustration shows the interior wavelet of the simplest type $p=1$. This wavelet has two vanishing moments $(r=2)$, and it belongs to $B_{q}^{\alpha}\left(L_{q}\right)$ for $(\alpha, q)$ with $\alpha<1+1 / q$. The corresponding wavelet system constitutes an $\alpha_{0}$-basis with $\alpha_{0}=2$.

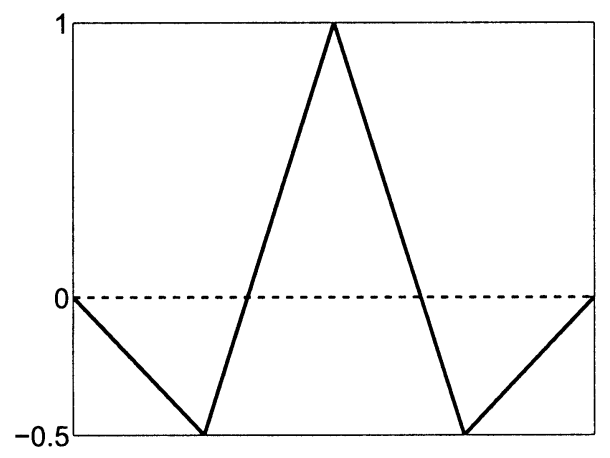


In the case that $\alpha$ and $q$ are related by $q^{-1}=\alpha+\frac{1}{2}$, the norm equivalence (2) simply reads

$$
\left\|\sum_{j, k} c_{j k} \psi_{j k}\right\|_{B_{q}^{\alpha}\left(L_{q}([0,1])\right)} \sim\left\|\left(c_{j k}\right)\right\|_{\ell_{q}(\mathbf{N})}, \quad 0<\alpha<\alpha_{0}, \quad q=\left(\alpha+\frac{1}{2}\right)^{-1} .
$$

Note that, for $\alpha>\frac{1}{2}$, the corresponding value of $q$ is between 0 and 1 . In this case we have to deal with the quasi-Banach spaces $\ell_{q}$ (for which the triangle inequality fails to hold).

By tensorization of the one-dimensional basis $\left\{\psi_{j k}\right\}$ we get a basis for $L_{2}\left([0,1]^{d}\right)$ :

$$
\begin{gathered}
\left\{\psi_{j_{1} k_{1} \cdots j_{d} k_{d}}: j_{i} \in \mathbf{N}, 1 \leq k_{i} \leq K_{j_{i}}\right\}, \\
\psi_{j_{1} k_{1} \cdots j_{d} k_{d}}(x):=\psi_{j_{1} k_{1}} \otimes \cdots \otimes \psi_{j_{d} k_{d}}(x)=\psi_{j_{1} k_{1}}\left(x_{1}\right) \times \cdots \times \psi_{j_{d} k_{d}}\left(x_{d}\right) .
\end{gathered}
$$

This basis is by construction anisotropic, that is, low frequencies in some directions can be paired with high frequencies in other directions. Certain finite subsets of this anisotropic tensor product basis are the so-called sparse grid spaces

$$
\hat{V}_{L}:=\left\{\psi_{j_{1} k_{1} \cdots j_{d} k_{d}}: j_{1}+\cdots+j_{d} \leq L, 1 \leq k_{i} \leq K_{j_{i}}\right\} .
$$

We aim at describing the approximation spaces for best $N$ term approximation in the multidimensional case by tensorization of the one-dimensional spaces. For this we first have to declare a tensor product structure on pairs of the involved spaces $B_{q}^{\alpha}\left(L_{q}\right)$ and $\ell_{q}$. This is done in the following section.

\section{A Tensor Product Structure on Certain Quasi-Banach Spaces}

For the later development, we need a tensor product structure on pairs of $B_{q}^{\alpha}\left(L_{q}\right)$ and $\ell_{q}$, respectively. If $q \geq 1$, we are in the realm of Banach spaces and such a structure is well known. We state this result for the spaces $\ell_{q}\left(\mathbf{N}^{d}\right)$ and $\ell_{q}(\mathbf{N})$.

Theorem 3. Let $q \geq 1$. Then there is a tensor norm $\|\cdot\|_{q}$ on the algebraic tensor product

$$
\ell_{q}\left(\mathbf{N}^{d}\right) \otimes \ell_{q}(\mathbf{N}) .
$$

We denote the completion of $\ell_{q}\left(\mathbf{N}^{d}\right) \otimes \ell_{q}(\mathbf{N})$ with respect to this norm by the tensor product $\ell_{q}\left(\mathbf{N}^{d}\right) \otimes_{q} \ell_{q}(\mathbf{N})$. This space is a Banach space, and the following isometry holds:

$$
\ell_{q}\left(\mathbf{N}^{d}\right) \otimes_{q} \ell_{q}(\mathbf{N}) \cong \ell_{q}\left(\mathbf{N}^{d+1}\right) .
$$

For a proof and more details, see, e.g., [18].

However, in the case $q<1$ such a structure does not seem to be known. We will give a tensor product structure for a special class of quasi-Banach spaces including $\ell_{q}$ and $B_{q}^{\alpha}\left(L_{q}\right)$. First, we have to introduce some notation. 
Definition 1. Let $X$ be a linear space. A function $\|\cdot\|_{X}: X \rightarrow \mathbf{R}$ is a $q$-quasi-norm $(0<q<1)$ if the following three properties hold:

(a) $\|x\|_{X}=0$ if and only if $x=0$;

(b) $\|\alpha x\|_{X}=|\alpha|\|x\|_{X}$ for $x \in X$ and $\alpha \in \mathbf{R}$; and

(c) $\|x+y\|_{X}^{q} \leq\|x\|_{X}^{q}+\|y\|_{X}^{q}$ for $x, y \in X$.

If $X$ is complete with respect to the quasi-metric induced by $\|\cdot\|_{X}$, we call $X$ a $q$-quasiBanach space.

Examples. For $0<q<1$, the spaces $\ell_{q}\left(\mathbf{N}^{d}\right), L_{q}(\Omega)$, and $B_{q}^{\alpha}\left(L_{q}(\Omega)\right)$ are $q$-quasiBanach spaces.

Remark 2. A $q$-quasi-Banach space, $0<q<1$, does not necessarily have a nontrivial topological dual. For instance, the topological dual of $L_{q}(\Omega)$, where $\Omega \subset \mathbf{R}^{d}$ is a domain and $0<q<1$, is trivial. However, the topological dual of $\ell_{q}\left(\mathbf{N}^{d}\right)$ is $\ell_{\infty}\left(\mathbf{N}^{d}\right)$ for any $0<q \leq 1$.

Definition 2. Let $X$ be a $q$-quasi-Banach space. We say $X$ is placid if there is a constant $C=C(X)$ such that for all finite sets $\left\{x, x_{1}, \ldots, x_{n}\right\} \subset X$ there exists a functional $\varphi$ (not necessarily continuous on all of $X$ ) with

$$
\varphi(x)=\|x\|_{X},
$$

and

$$
\sum_{i=1}^{n}\left|\varphi\left(x_{i}\right)\right|^{q} \leq C \sum_{i=1}^{n}\left\|x_{i}\right\|_{X}^{q}
$$

The functional $\varphi$ in the above definition is a type of substitute for the Hahn-Banach extension theorem, which generally fails in quasi-Banach spaces due to nonconvexity. We now show that $q$-quasi-Banach spaces which admit a norm-equivalence to $\ell_{q}$ are placid.

Definition 3. A $q$-quasi-Banach space $X$ admits a $q$-estimate (for $0<q<1$ ) if there is a set of functions $\left\{f_{i}\right\}$ spanning $X$, such that

$$
\left\|\sum_{i} c_{i} f_{i}\right\|_{X} \sim\left\|\left(c_{i}\right)\right\|_{\ell_{q}(\mathbf{N})}
$$

for all convergent series $\sum_{i} c_{i} f_{i}$.

Examples. The spaces $B_{q}^{\alpha}\left(L_{q}([0,1])\right)$ (with $\left.q^{-1}=\alpha+\frac{1}{2}\right)$ admit $q$-estimates.

Lemma 1. Let $X$ be a q-quasi-Banach space admitting a q-estimate. Then $X$ is placid. In particular, $\ell_{q}\left(\mathbf{N}^{d}\right)$ and $B_{q}^{\alpha}\left(L_{q}(\Omega)\right.$ ) (with $q^{-1}=\alpha+\frac{1}{2}$ ) are placid. In the case of $X=\ell_{q}\left(\mathbf{N}^{d}\right)$, the constant $C$ in the definition of "placid" can be chosen to be one. 
Proof. We begin with the case $X=\ell_{q}\left(\mathbf{N}^{d}\right)$. We identify the topological dual of $\ell_{q}\left(\mathbf{N}^{d}\right)$ in the standard way with $\ell_{\infty}\left(\mathbf{N}^{d}\right)$.

Fix $x, x_{1}, \ldots, x_{n} \in X$ for some $n \in \mathbf{N}$. Let $e_{j_{1} \ldots j_{d}}$ be the evaluation functional of the $\left(j_{1}, \ldots, j_{d}\right)$ th component:

$$
e_{j_{1} \cdots j_{d}}(y)=y^{j_{1} \cdots j_{d}}, \quad y=\left(y^{i_{1} \cdots i_{d}}\right)_{\left(i_{1}, \ldots, i_{d}\right) \in \mathbf{N}^{d}} \in \ell_{q}\left(\mathbf{N}^{d}\right) .
$$

Now set

$$
C_{j_{1} \cdots j_{d}}:=\left(\frac{\sum_{i=1}^{n}\left\|x_{i}\right\|_{\ell_{q}\left(\mathbf{N}^{d}\right)}^{q}}{\sum_{i=1}^{n}\left|x_{i}^{j_{1} \cdots j_{d}}\right|^{q}}\right)^{1 / q}
$$

(these numbers being finite) and consider the set of functionals

$$
A=\left\{\varphi_{j_{1} \cdots j_{d}}=\left(\operatorname{sgn} x^{j_{1} \cdots j_{d}}\right) C_{j_{1} \cdots j_{d}} e_{j_{1} \cdots j_{d}}:\left(j_{1}, \ldots, j_{d}\right) \in \mathbf{N}^{d}\right\} \subset\left(\ell_{q}\left(\mathbf{N}^{d}\right)\right)^{\prime} .
$$

Note that

$$
\varphi \in A \Rightarrow \sum_{i=1}^{n}\left|\varphi\left(x_{i}\right)\right|^{q}=\sum_{i=1}^{n}\left\|x_{i}\right\|_{\ell_{q}\left(\mathbf{N}^{d}\right)}^{q} .
$$

Claim. There is $\varphi \in A$ such that $\varphi(x) \geq\|x\|_{\ell_{q}\left(\mathbf{N}^{d}\right)}$. Downscaling then yields the assertion of the lemma.

Observe that

$$
\sum_{j_{1}, \ldots, j_{d}=1}^{\infty} C_{j_{1} \cdots j_{d}}^{-q}=\sum_{j_{1}, \ldots j_{d}=1}^{\infty} \frac{\sum_{i=1}^{n}\left|x_{i}^{j_{1} \cdots j_{d}}\right|^{q}}{\sum_{i=1}^{n}\left\|x_{i}\right\|_{\ell_{q}\left(\mathbf{N}^{d}\right)}^{q}}=1 .
$$

Now assume that there is no $\varphi \in A$ with $\varphi(x) \geq\|x\|_{\ell_{q}\left(\mathbf{N}^{d}\right)}$. This implies

$$
C_{j_{1} \cdots j_{d}}<\frac{\|x\|_{\ell_{q}\left(\mathbf{N}^{d}\right)}}{\left|x^{j_{1} \cdots j_{d}}\right|} \quad \text { for all } \quad\left(j_{1}, \ldots, j_{d}\right) \in \mathbf{N}^{d}
$$

Summing up gives

$$
\sum_{j_{1}, \ldots, j_{d}=1}^{\infty} C_{j_{1} \cdots j_{d}}^{-q}>1
$$

this is a contradiction.

In the general case we use the $q$-estimate to transfer the problem to $\ell_{q}$ : write $x=$ $\sum_{k} c_{k} f_{k}$ and $x_{i}=\sum_{k} c_{k}^{i} f_{k}$. Then choose $\tilde{\varphi} \in \ell_{q}(\mathbf{N})^{\prime}$ such that $\tilde{\varphi}\left(\left(c_{k}\right)\right)=\left\|\left(c_{k}\right)\right\|_{\ell_{q}(\mathbf{N})}$ and $\sum_{i=1}^{n}\left|\tilde{\varphi}\left(\left(c_{k}^{i}\right)_{k}\right)\right|^{q} \leq \sum_{i=1}^{n}\left\|\left(c_{k}^{i}\right)_{k}\right\|_{\ell_{q}(\mathbf{N})}^{q}$. Employing the $q$-estimate and rescaling of $\tilde{\varphi}$ yields the assertion with $C$ the quotient of the lower and upper constants in the $q$-estimate to the power $q$.

Remark 3. It is not always possible to have $C=1$ for the constant in the definition of "placid." To see this, consider the two-dimensional linear space $Q$ spannend by two 
elements $u$ and $v$ together with the function

$$
\|\cdot\|: Q \rightarrow \mathbf{R}_{+}, \quad\|a u+b v\|= \begin{cases}a+(s-1) b & \text { if } 0 \leq b \leq a \\ (s-1) a+b & \text { if } 0 \leq a \leq b, \\ -3 a+b & \text { if } a \leq 0 \leq b \text { and }|a| \leq b \\ -a+3 b & \text { if } a \leq 0 \leq b \text { and }|a| \geq b\end{cases}
$$

for $b \geq 0$ and $\|a u+b v\|=\|-a u-b v\|$ for $b \leq 0$; here $s=(2-\sqrt{2})^{2}$.

The function $\|\cdot\|$ is a 0.5 -quasi-norm on $Q$. However, for $x_{1}=u, x_{2}=v, x_{3}=u+v$, and $x=u-v$, we have

$$
\min _{\varphi: \varphi(x)=\|x\|} \sum_{i=1}^{3}\left|\varphi\left(x_{i}\right)\right|^{0.5}=2^{1.5} \approx 2.83>\sum_{i=1}^{3}\left\|x_{i}\right\|^{0.5}=4-\sqrt{2} \approx 2.59 .
$$

Remark 4. We conjecture that not every $q$-quasi-Banach space is placid. However, the spaces of interest to us, $\ell_{q}\left(\mathbf{N}^{d}\right)$ and $B_{q}^{\alpha}\left(L_{q}(\Omega)\right)\left(q^{-1}=\alpha+\frac{1}{2}\right)$, are placid.

We further need several more $\ell_{q}$-type spaces:

(a) $\ell_{q}(n)$ is the space of all $n$-vectors

$$
x=\left(x^{1}, \ldots, x^{n}\right),
$$

for which

$$
\|x\|_{\ell_{q}(n)}:=\left(\sum_{i=1}^{n}\left|x^{i}\right|^{q}\right)^{1 / q}
$$

is finite.

(b) $\ell_{q}(n, X)$ is the space of all $X$-valued $n$-vectors ( $X$ a $q$-quasi-Banach space)

$$
x=\left(x^{1}, \ldots, x^{n}\right), \quad x^{i} \in X,
$$

for which

$$
\|x\|_{\ell_{q}(n, X)}:=\left(\sum_{i=1}^{n}\left\|x^{i}\right\|_{X}^{q}\right)^{1 / q}
$$

is finite.

(c) $\ell_{q}\left(\mathbf{N}^{n}, X\right.$ ) is the space of all $X$-valued $n$-multi-indexed sequences ( $X$ is a $q$-quasiBanach space)

$$
x=\left(x^{i_{1} \cdots i_{n}}\right)_{\left(i_{1}, \ldots, i_{n}\right) \in \mathbf{N}^{n}}, \quad x^{i_{1}, \ldots, i_{n}} \in X,
$$

for which

$$
\|x\|_{\ell_{q}\left(\mathbf{N}^{n}, X\right)}:=\left(\sum_{i_{1}, \ldots, i_{n}=1}^{\infty}\left\|x^{i_{1} \cdots i_{n}}\right\|_{X}^{q}\right)^{1 / q}
$$

is finite. 
Now we have the notation to declare a topological tensor product structure on the algebraic tensor products of placid $q$-quasi-Banach spaces. This is done in the following theorem.

Theorem 4. Let $X$ and $Y$ be placid q-quasi-Banach spaces, $0<q<1$. Then the function

$$
\begin{gathered}
\|\cdot\|: X \otimes Y \rightarrow \mathbf{R}, \\
\|z\|:=\inf _{n \in \mathbf{N}, z=\sum_{i=1}^{n} x_{i} \otimes y_{i}}\left\|\left(x_{i}\right)\right\|_{\ell_{q}(n, X)} \sup _{\|\lambda\|_{\ell(n)} \leq 1}\left\|\sum_{i=1}^{n} \lambda_{i} y_{i}\right\|_{Y},
\end{gathered}
$$

on the algebraic tensor product of $X$ and $Y$ is a q-quasi-norm. We denote the completion of the algebraic tensor product $X \otimes Y$ under the induced quasi-metric by $X \otimes_{q} Y$ and call it the q-tensor product of $X$ and $Y$.

In the case of $X=\ell_{q}\left(\mathbf{N}^{d}\right)$ and $Y=\ell_{q}(\mathbf{N})$, the function $\|\cdot\|$ is a cross-norm, i.e., for simple tensor products $x \otimes y$ it holds that $\|x \otimes y\|=\|x\|_{\ell_{q}\left(\mathbf{N}^{d}\right)}\|y\|_{\ell_{q}(\mathbf{N})}$. Furthermore, we have an isometric isomorphism

$$
\ell_{q}\left(\mathbf{N}^{d}\right) \otimes_{q} \ell_{q}(\mathbf{N}) \cong \ell_{q}\left(\mathbf{N}^{d+1}\right) .
$$

Remark 5. The tensor product structure and the isometry result, as stated in Theorem 4, seem to be new. The proof relies on a type of substitute for the Hahn-Banach extension theorem valid for finite-dimensional subspaces of placid $q$-quasi-Banach spaces, see Definition 2 and Lemma 1. The Hahn-Banach extension theorem fails in general in quasiBanach spaces, due to nonconvexity. For an account of the state of the art knowledge on quasi-Banach spaces, see [17].

Remark 6. We are aware of the result of Turpin [27] on tensor products of $q$-quasiBanach spaces. In the paper cited it was proven that if $0<p, q \leq 1, X$ is a $p$-quasiBanach space and $Y$ is a $q$-quasi-Banach space, then there is a tensor product structure on $X \otimes Y$ such that $X \otimes Y$ is an $r$-quasi-Banach space, provided

$$
\frac{1}{r} \geq \frac{1}{p}+\frac{1}{q}-1
$$

For $p=q<1$ this implies $r \leq q /(2-q)<q$, which is not sufficient for our purpose.

Proof of Theorem 4. We first note that, by definition of the (algebraic) tensor product, we have

$$
\sum_{i=1}^{n} x_{i} \otimes y_{i}=\sum_{i=1}^{\bar{n}} \bar{x}_{i} \otimes \bar{y}_{i}, \quad x_{i}, \bar{x}_{i} \in X, \quad y_{i}, \bar{y}_{i} \in Y,
$$

if and only if

$$
\text { for all } \varphi \in X^{*}, \quad \sum_{i=1}^{n} \varphi\left(x_{i}\right) y_{i}=\sum_{i=1}^{\bar{n}} \varphi\left(\bar{x}_{i}\right) \bar{y}_{i} .
$$


Here we denote by $X^{*}$ the (always nontrivial!) algebraic dual of the $q$-quasi-Banach space $X$.

We begin with the first part of the statement of Theorem 4 . We have to show properties (a), (b), (c) of the definition of a $q$-quasi-norm.

Property (b), i.e., $\|\alpha z\|=|\alpha|\|z\|$ for $z \in X \otimes Y, \alpha \in \mathbf{R}$, readily follows, since the involved quasi-norms are homogeneous.

Property (a), i.e., $\|z\|=0$ if and only if $z=0$.

If $z=0=0 \otimes 0$, we have $\|z\|=0$. On the contrary, assume $z \neq 0$. Let $z=$ $\sum_{i=1}^{\bar{n}} \bar{x}_{i} \otimes \bar{y}_{i}$ be a representation of $z$ with linearly independent sets $\left(\bar{x}_{i}\right),\left(\bar{y}_{i}\right)$. (Such a linearly independent representation always exists for every algebraic tensor product.)

Fix one of the pairs $\left(\bar{x}_{i}, \bar{y}_{i}\right)$, say $\left(\bar{x}_{1}, \bar{y}_{1}\right)$, and set

$$
\varepsilon:=\min _{\alpha_{i}}\left\{\|y\|_{Y}: y=\left\|\bar{x}_{1}\right\|_{X} \bar{y}_{1}+\sum_{i=2}^{\bar{n}} \alpha_{i} \bar{y}_{i}\right\}>0 .
$$

Now choose, according to Lemma 1, a functional $\varphi$ with

$$
\varphi\left(\bar{x}_{1}\right)=\left\|\bar{x}_{1}\right\|_{X} \quad \text { and } \quad \sum_{i=1}^{\bar{n}}\left|\varphi\left(\bar{x}_{i}\right)\right|^{q} \leq C \sum_{i=1}^{\bar{n}}\left\|\bar{x}_{i}\right\|_{X}^{q} .
$$

Let an arbitrary representation $\sum_{i=1}^{n} x_{i} \otimes y_{i}$ of $z$ be given. Then we estimate

$$
\begin{aligned}
\left\|\sum_{i=1}^{n} \varphi\left(x_{i}\right) y_{i}\right\|_{Y}^{q} & \leq \sum_{i=1}^{n}\left|\varphi\left(x_{i}\right)\right|^{q}\left\|y_{i}\right\|_{Y}^{q} \leq C \sum_{i=1}^{n}\left\|x_{i}\right\|_{X}^{q} \sup _{i=1, \ldots, n}\left\|y_{i}\right\|_{Y}^{q} \\
& \leq C\left\|\left(x_{i}\right)\right\|_{\ell_{q}(n, X)}^{q} \sup _{\|\lambda\|_{\ell_{q}(n)} \leq 1}\left\|\sum_{i=1}^{n} \lambda_{i} y_{i}\right\|_{Y}^{q}
\end{aligned}
$$

On the other hand, we have

$$
\begin{aligned}
\left\|\sum_{i=1}^{n} \varphi\left(x_{i}\right) y_{i}\right\|_{Y}^{q} & =\left\|\sum_{i=1}^{\bar{n}} \varphi\left(\bar{x}_{i}\right) \bar{y}_{i}\right\|_{X}^{q} \\
& =\|\| \bar{x}_{1}\left\|_{X} \bar{y}_{1}+\sum_{i=2}^{\bar{n}} \varphi\left(\bar{x}_{i}\right) \bar{y}_{i}\right\|_{X}^{q} \geq \varepsilon^{q} .
\end{aligned}
$$

Combining this gives

$$
\varepsilon C^{-1 / q} \leq\left\|\left(x_{i}\right)\right\|_{\ell_{q}(n, X)} \sup _{\|\lambda\|_{\ell_{q}(n)} \leq 1}\left\|\sum_{i=1}^{n} \lambda_{i} y_{i}\right\|_{Y}
$$

for every representation of $z$. Taking the infimum over all representations yields $\|z\| \geq$ $\varepsilon C^{-1 / q}>0$.

Property (c), i.e., the generalized triangle inequality $\|z+w\|^{q} \leq\|z\|^{q}+\|w\|^{q}$. 
We show first

$$
\left\|\sum_{i=1}^{n} \mu_{i} y_{i}\right\|_{Y}^{q} \leq\|\mu\|_{\ell_{q}(n)}^{q} \sup _{\|\lambda\|_{\ell_{q}(n)} \leq 1}\left\|\sum_{i=1}^{n} \lambda_{i} y_{i}\right\|_{Y}^{q} .
$$

For this purpose set $\theta:=\|\mu\|_{\ell_{q}(n)}^{-1}$, such that $\|\theta \mu\|_{\ell_{q}(n)}=1$. Then we have

$$
\begin{aligned}
\left\|\sum_{i=1}^{n} \mu_{i} y_{i}\right\|_{Y}^{q} & =\theta^{-q}\left\|\sum_{i=1}^{n} \theta \mu_{i} y_{i}\right\|_{Y}^{q}=\|\mu\|_{\ell_{q}(n)}^{q}\left\|\sum_{i=1}^{n} \theta \mu_{i} y_{i}\right\|_{Y}^{q} \\
& \leq\|\mu\|_{\ell_{q}(n)}^{q} \sup _{\|\lambda\|_{\ell_{q}(n)} \leq 1}\left\|\sum_{i=1}^{n} \lambda_{i} y_{i}\right\|_{Y}^{q} .
\end{aligned}
$$

Next we show that, for $m<n$,

$$
\sup _{\|\lambda\|_{e_{q}(m)} \leq 1}\left\|\sum_{i=1}^{m} \lambda_{i} y_{i}\right\|_{Y}^{q} \leq 1 \quad \text { and } \quad \sup _{\|\lambda\|_{\ell_{q}(n-m)} \leq 1}\left\|\sum_{i=m+1}^{n} \lambda_{i-m} y_{i}\right\|_{Y}^{q} \leq 1,
$$

implies

$$
\sup _{\|\lambda\|_{e_{q}(n)} \leq 1}\left\|\sum_{i=1}^{n} \lambda_{i} y_{i}\right\|_{Y}^{q} \leq 1
$$

To this end, let $\lambda \in \ell_{q}(n)$ with $\|\lambda\|_{\ell_{q}(n)} \leq 1$. We write $\lambda=\mu+\sigma$ with

$$
\mu=\left(\lambda_{1}, \ldots, \lambda_{m}\right) \quad \text { and } \quad \sigma=\left(\lambda_{m+1}, \ldots, \lambda_{n}\right) .
$$

Then it holds that $\|\mu\|_{\ell_{q}(n)}^{q}+\|\sigma\|_{\ell_{q}(n)}^{q}=\|\lambda\|_{\ell_{q}(n)}^{q} \leq 1$. Using (5), we get

$$
\begin{aligned}
\left\|\sum_{i=1}^{n} \lambda_{i} y_{i}\right\|_{Y}^{q} \leq & \left\|\sum_{i=1}^{m} \lambda_{i} y_{i}\right\|_{Y}^{q}+\left\|\sum_{i=m+1}^{n} \lambda_{i} y_{i}\right\|_{Y}^{q} \\
\leq & \|\mu\|_{\ell_{q}(m)}^{q} \sup _{\|\kappa\|_{\ell_{q}(m)} \leq 1}\left\|\sum_{i=1}^{m} \kappa_{i} y_{i}\right\|_{Y}^{q} \\
& +\|\sigma\|_{\ell_{q}(n-m)}^{q} \sup _{\|\kappa\|_{\ell_{q}(n-m)} \leq 1}\left\|\sum_{i=m+1}^{n} \kappa_{i-m} y_{i}\right\|_{Y}^{q} .
\end{aligned}
$$

Using (6) and $\|\mu\|_{\ell_{q}(n)}^{q}+\|\sigma\|_{\ell_{q}(n)}^{q}=\|\lambda\|_{\ell_{q}(n)}^{q} \leq 1$, it follows that

$$
\left\|\sum_{i=1}^{n} \lambda_{i} y_{i}\right\|_{Y}^{q} \leq 1
$$

Taking the supremum over all $\lambda$ with $\|\lambda\|_{\ell_{q}(n)} \leq 1$ gives (7).

Now let $z, w \in X \otimes Y$, and let $\varepsilon>0$. Choose a representation $z=\sum_{i=1}^{m} x_{i} \otimes y_{i}$ with

$$
\left\|\left(x_{i}\right)\right\|_{\ell_{q}(m, X)} \sup _{\|\lambda\|_{\ell_{q}(m) \leq 1}}\left\|\sum_{i=1}^{m} \lambda_{i} y_{i}\right\|_{Y} \leq\left(\|z\|^{q}+\varepsilon\right)^{1 / q} .
$$


Due to the homogeneity of the tensor product, we can assume, without loss of generality,

$$
\left\|\left(x_{i}\right)\right\|_{\ell_{q}(m, X)}^{q} \leq\|z\|^{q}+\varepsilon, \quad \sup _{\|\lambda\|_{\ell_{q}(m)} \leq 1}\left\|\sum_{i=1}^{m} \lambda_{i} y_{i}\right\|_{Y}^{q} \leq 1 .
$$

Analogously, we choose a representation $w=\sum_{i=m+1}^{n} x_{i} \otimes y_{i}$ with

$$
\left\|\left(x_{m+i}\right)\right\|_{\ell_{q}(n-m, X)}^{q} \leq\|w\|^{q}+\varepsilon, \quad \sup _{\|\lambda\|_{\ell_{q}(n-m)} \leq 1}\left\|\sum_{i=m+1}^{n} \lambda_{i-m} y_{i}\right\|_{Y}^{q} \leq 1 .
$$

Then, with (7),

$$
\begin{aligned}
\left\|\left(x_{i}\right)\right\|_{\ell_{q}(n, X)}^{q} \sup _{\|\lambda\|_{\ell_{q}(n) \leq 1} \|}\left\|\sum_{i=1}^{n} \lambda_{i} y_{i}\right\|_{Y}^{q} & \leq\left(\left\|\left(x_{i}\right)\right\|_{\ell_{q}(m, X)}^{q}+\left\|\left(x_{m+i}\right)\right\|_{\ell_{q}(n-m, X)}^{q}\right) \cdot 1 \\
& \leq\|z\|^{q}+\|w\|^{q}+2 \varepsilon .
\end{aligned}
$$

Since $z+w=\sum_{i=1}^{n} x_{i} \otimes y_{i}$ is a representation of $z+w$, we infer

$$
\|z+w\|^{q} \leq\|z\|^{q}+\|w\|^{q}+2 \varepsilon .
$$

Sending $\varepsilon \rightarrow 0$, we arrive at the generalized triangle inequality. This proves the first part of Theorem 4.

For the second part, we first show that the $q$-quasi-norm on $\ell_{q}\left(\mathbf{N}^{d}\right) \otimes_{q} \ell_{q}(\mathbf{N})$ is a cross-norm, i.e., for simple tensors $x \otimes y$ it holds that $\|x \otimes y\|=\|x\|_{\ell_{q}\left(\mathbf{N}^{d}\right)}\|y\|_{\ell_{q}(\mathbf{N})}$. Let $x \otimes y=\sum_{i=1}^{n} x_{i} \otimes y_{i}$ be any representation of $x \otimes y$. According to Lemma 1 choose a functional $\varphi$ such that

$$
\varphi(x)=\|x\|_{\ell_{q}\left(\mathbf{N}^{d}\right)} \quad \text { and } \quad \sum_{i=1}^{n}\left|\varphi\left(x_{i}\right)\right|^{q} \leq \sum_{i=1}^{n}\left\|x_{i}\right\|_{\ell_{q}\left(\mathbf{N}^{d}\right)}^{q} .
$$

Now set $M=\left(\sum_{i=1}^{n}\left|\varphi\left(x_{i}\right)\right|^{q}\right)^{1 / q}$ and $\lambda_{i}=\varphi\left(x_{i}\right) / M$. Then, $\|\lambda\|_{\ell_{q}(n)}=1$. We have

$$
\begin{aligned}
\left\|\left(x_{i}\right)\right\|_{\ell_{q}\left(n, \ell_{q}\left(\mathbf{N}^{d}\right)\right)} \sup _{\|\mu\|_{\ell_{q}(n)}=1}\left\|\sum_{i=1}^{n} \mu_{i} y_{i}\right\|_{\ell_{q}(\mathbf{N})} & \geq\left\|\left(x_{i}\right)\right\|_{\ell_{q}\left(n, \ell_{q}\left(\mathbf{N}^{d}\right)\right)}\left\|\sum_{i=1}^{n} \lambda_{i} y_{i}\right\|_{\ell_{q}(\mathbf{N})} \\
= & \left(\sum_{i=1}^{n}\left\|x_{i}\right\|_{\ell_{q}\left(\mathbf{N}^{d}\right)}^{q}\right)^{1 / q} \cdot \frac{1}{M}\left\|\sum_{i=1}^{n} \varphi\left(x_{i}\right) y_{i}\right\|_{\ell_{q}(\mathbf{N})} \\
= & \left(\frac{\left.\sum_{i=1}^{n}\left\|x_{i}\right\|_{\ell_{q}}^{q} \mathbf{N}^{d}\right)}{\sum_{i=1}^{n} \mid \varphi\left(x_{i}\right)^{q}}\right)^{1 / q} \\
& \times\|\varphi(x) y\|_{\ell_{q}(\mathbf{N})} \geq\|x\|_{\ell_{q}\left(\mathbf{N}^{d}\right)}\|y\|_{\ell_{q}(\mathbf{N}) .}
\end{aligned}
$$

Taking the infimum over all representations of $x \otimes y$ yields

$$
\|x \otimes y\| \geq\|x\|_{\ell_{q}\left(\mathbf{N}^{d}\right)}\|y\|_{\ell_{q}(\mathbf{N})} .
$$


The reverse inequality $\|x \otimes y\| \leq\|x\|_{\ell_{q}\left(\mathbf{N}^{d}\right)}\|y\|_{\ell_{q}(\mathbf{N})}$ is trivial by the definition of $\|\cdot\|$.

Finally, we show the isometry $\ell_{q}\left(\mathbf{N}^{d}\right) \otimes_{q} \ell_{q}(\mathbf{N}) \cong \ell_{q}\left(\mathbf{N}^{d}, \ell_{q}(\mathbf{N})\right)$. The remaining isometry $\ell_{q}\left(\mathbf{N}^{d}, \ell_{q}(\mathbf{N})\right) \cong \ell_{q}\left(\mathbf{N}^{d+1}\right)$ is standard.

Let us first consider the mapping

$$
\begin{gathered}
\Lambda: \ell_{q}\left(\mathbf{N}^{d}\right) \otimes \ell_{q}(\mathbf{N}) \rightarrow \ell_{q}\left(\mathbf{N}^{d}, \ell_{q}(\mathbf{N})\right), \\
z=\sum_{i=1}^{n} x_{i} \otimes y_{i} \mapsto\left[f:\left(j_{1}, \ldots, j_{d}\right) \mapsto \sum_{i=1}^{n} x_{i}^{j_{1} \cdots j_{d}} y_{i}\right] .
\end{gathered}
$$

The mapping $\Lambda$ is well defined. To see this, take two representations

$$
z=\sum_{i=1}^{n} x_{i} \otimes y_{i}=\sum_{i=1}^{\bar{n}} \bar{x}_{i} \otimes \bar{y}_{i} \in \ell_{q}\left(\mathbf{N}^{d}\right) \otimes \ell_{q}(\mathbf{N})
$$

and denote

$$
f:\left(j_{1}, \ldots, j_{d}\right) \mapsto \sum_{i=1}^{n} x_{i}^{j_{1} \cdots j_{d}} y_{i}, \quad \bar{f}:\left(j_{1}, \ldots, j_{d}\right) \mapsto \sum_{i=1}^{\bar{n}} \bar{x}_{i}^{j_{1} \cdots j_{d}} \bar{y}_{i} .
$$

Let $e_{j_{1} \cdots j_{d}}$ be the evaluation functional of the $\left(j_{1}, \ldots, j_{d}\right)$ th component:

$$
e_{j_{1} \cdots j_{d}}(x)=x^{j_{1} \cdots j_{d}}, \quad x=\left(x^{i_{1} \cdots i_{d}}\right)_{\left(i_{1}, \ldots, i_{d}\right) \in \mathbf{N}^{d}} \in \ell_{q}\left(\mathbf{N}^{d}\right) .
$$

Then we have

$$
\begin{aligned}
f\left(\left(j_{1}, \ldots, j_{d}\right)\right) & =\sum_{i=1}^{n} x_{i}^{j_{1} \cdots j_{d}} y_{i}=\sum_{i=1}^{n} e_{j_{1} \cdots j_{d}}\left(x_{i}\right) y_{i} \\
& =\sum_{i=1}^{\bar{n}} e_{j_{1} \cdots j_{d}}\left(\bar{x}_{i}\right) \bar{y}_{i}=\sum_{i=1}^{\bar{n}} \bar{x}_{i}^{j_{1} \cdots j_{d}} \bar{y}_{i}=\bar{f}\left(\left(j_{1}, \ldots, j_{d}\right)\right)
\end{aligned}
$$

hence $f=\bar{f}$.

Now we show that $\Lambda$ is of norm 1: Let $z \in \ell_{q}\left(\mathbf{N}^{d}\right) \otimes \ell_{q}(\mathbf{N})$ and $f=\Lambda(z)$. For an arbitrary representation $z=\sum_{i=1}^{n} x_{i} \otimes y_{i}$, we have

$$
\begin{aligned}
\|f\|_{\ell_{q}\left(\mathbf{N}^{d}, \ell_{q}(\mathbf{N})\right)}^{q} & =\sum_{j_{1}, \ldots, j_{d}}^{\infty}\left\|\sum_{i=1}^{n} x_{i}^{j_{1} \cdots j_{d}} y_{i}\right\|_{\ell_{q}(\mathbf{N})}^{q} \leq \sum_{j_{1}, \ldots, j_{d}}^{\infty} \sum_{i=1}^{n}\left|x_{i}^{j_{1} \cdots j_{d}}\right|^{q}\left\|y_{i}\right\|_{\ell_{q}(\mathbf{N})}^{q} \\
& =\sum_{i=1}^{n}\left\|x_{i}\right\|_{\ell_{q}\left(\mathbf{N}^{d}\right)}^{q}\left\|y_{i}\right\|_{\ell_{q}(\mathbf{N})}^{q} \leq \sum_{i=1}^{n}\left\|x_{i}\right\|_{\ell_{q}\left(\mathbf{N}^{d}\right)}^{q} \max _{i=1, \ldots, n}\left\|y_{i}\right\|_{\ell_{q}(\mathbf{N})}^{q} \\
& \leq\left\|\left(x_{i}\right)\right\|_{\ell_{q}\left(n, \ell_{q}\left(\mathbf{N}^{d}\right)\right)}^{q}\left(\sup _{\|\lambda\|_{\ell_{q}(n)} \leq 1}\left\|\sum_{i=1}^{n} \lambda_{i} y_{i}\right\|_{\ell_{q}(\mathbf{N})}\right)^{q} .
\end{aligned}
$$

Taking the infimum over all representations on the right, we arrive at

$$
\|f\|_{\ell_{q}\left(\mathbf{N}^{d}, \ell_{q}(\mathbf{N})\right)}^{q} \leq\|z\|^{q} .
$$


Thus, $\Lambda$ has norm $\leq 1$. To see the equality, take a simple tensor $x \otimes y \in \ell_{q}\left(\mathbf{N}^{d}\right) \otimes \ell_{q}(\mathbf{N})$. Then we have

$$
\begin{aligned}
\|f\|_{\ell_{q}\left(\mathbf{N}^{d}, \ell_{q}(\mathbf{N})\right)}^{q} & =\sum_{j_{1}, \ldots, j_{d}}^{\infty}\left\|x^{j_{1} \cdots j_{d}} y\right\|_{\ell_{q}(\mathbf{N})}^{q}=\sum_{j_{1}, \ldots, j_{d}}^{\infty}\left|x^{j_{1} \cdots j_{d}}\right|^{q}\|y\|_{\ell_{q}(\mathbf{N})}^{q} \\
& =\|x\|_{\ell_{q}\left(\mathbf{N}^{d}\right)}^{q}\|y\|_{\ell_{q}(\mathbf{N})}^{q}=\|x \otimes y\| .
\end{aligned}
$$

Thus, $\Lambda$ has norm 1 and gives, by continuous extension to $\ell_{q}\left(\mathbf{N}^{d}\right) \otimes_{q} \ell_{q}(\mathbf{N})$, an operator

$$
\tilde{\Lambda}: \ell_{q}\left(\mathbf{N}^{d}\right) \otimes_{q} \ell_{q}(\mathbf{N}) \rightarrow \ell_{q}\left(\mathbf{N}^{d}, \ell_{q}(\mathbf{N})\right)
$$

of norm 1.

Next, consider the mapping

$$
\tilde{\Gamma}: \ell_{q}\left(\mathbf{N}^{d}, \ell_{q}(\mathbf{N})\right) \rightarrow \ell_{q}\left(\mathbf{N}^{d}\right) \otimes_{q} \ell_{q}(\mathbf{N}),
$$

which is the continuous extension of the mapping $\Gamma$ defined on simple functions (i.e., functions of finite support)

$$
f:\{1, \ldots, n\}^{d} \rightarrow \ell_{q}(\mathbf{N}), \quad\left(j_{1}, \ldots, j_{d}\right) \mapsto c^{j_{1} \cdots j_{d}} \in \ell_{q}(\mathbf{N}) .
$$

With such a simple function, we associate

$$
z=\Gamma(f)=\sum_{\left(j_{1}, \ldots, j_{d}\right) \in\{1, \ldots, n\}^{d}} \underbrace{\chi^{j_{1} \cdots j_{d}}\left\|c^{j_{1} \cdots j_{d}}\right\|_{\ell_{q}(\mathbf{N})}}_{x^{j_{1} \cdots j_{l}}} \otimes \underbrace{\frac{c^{j_{1} \cdots j_{d}}}{\left\|c^{j_{1} \cdots j_{d}}\right\|_{\ell_{q}(\mathbf{N})}}}_{y^{j_{1} \cdots j_{d}}},
$$

where $\chi^{j_{1} \cdots j_{d}}$ denotes the characteristic function of the multi-index $\left(j_{1}, \ldots, j_{d}\right)$.

We show that $\Gamma$ is of norm 1 :

$$
\begin{aligned}
\|z\|^{q} & \leq\left(\sum_{\{1, \ldots, n\}^{d}}\left\|c^{j_{1} \cdots j_{d}}\right\|_{\ell_{q}(\mathbf{N})}^{q}\right) \underbrace{\sup _{\|\lambda\|_{\ell_{q}\left(n^{d}\right)} \leq 1}\left\|\sum_{\{1, \ldots, n\}^{d}} \lambda_{i} \frac{c^{j_{1} \cdots j_{d}}}{\left\|c^{j_{1} \cdots j_{d}}\right\|_{\ell_{q}(\mathbf{N})}}\right\|^{q}}_{\leq 1} \\
& \leq \sum_{\{1, \ldots, n\}^{d}}\left\|c^{j_{1} \cdots j_{d}}\right\|_{\ell_{q}(\mathbf{N})}^{q}=\|f\|_{\ell_{q}\left(\mathbf{N}^{d}, \ell_{q}(\mathbf{N})\right)}^{q} .
\end{aligned}
$$

Choosing $f=\chi^{j_{1} \cdots j_{d}} c^{j_{1} \cdots j_{d}}$ with $c^{j_{1} \cdots j_{d}} \in \ell_{q}(\mathbf{N})$ and norm 1 , we see that $\|z\|=1=\|f\|$. Hence, $\Gamma$ as well as the continuous extension $\tilde{\Gamma}$ are of norm 1 .

Now consider $\tilde{\Gamma} \tilde{\Lambda}: \ell_{q}\left(\mathbf{N}^{d}\right) \otimes_{q} \ell_{q}(\mathbf{N}) \rightarrow \ell_{q}\left(\mathbf{N}^{d}\right) \otimes_{q} \ell_{q}(\mathbf{N})$. This mapping has the norm $\leq 1$. We show that, for $z=\sum_{i=1}^{n} x_{i} \otimes y_{i}$ with $\operatorname{supp}\left(x_{i}\right) \subset\{1, \ldots, N\}^{d}$, this mapping is the identity

$$
\begin{aligned}
\tilde{\Gamma} \tilde{\Lambda}\left(\sum_{i=1}^{n} x_{i} \otimes y_{i}\right) & =\tilde{\Gamma}\left(\left(j_{1}, \ldots, j_{d}\right) \mapsto \sum_{i=1}^{n} x_{i}^{j_{1} \cdots j_{d}} y_{i}\right) \\
& =\sum_{\{1, \ldots, N\}^{d}} \chi^{j_{1} \cdots j_{d}}\left\|\sum_{i=1}^{n} x_{i}^{j_{1} \cdots j_{d}} y_{i}\right\|_{\ell_{q}(\mathbf{N})} \otimes \frac{\sum_{i=1}^{n} x_{i}^{j_{1} \cdots j_{d}} y_{i}}{\left\|\sum_{i=1}^{n} x_{i}^{j_{1} \cdots j_{d}} y_{i}\right\|_{\ell_{q}(\mathbf{N})}} \\
& =\sum_{\{1, \ldots, N\}^{d}} \chi^{j_{1} \cdots j_{d}} \otimes \sum_{i=1}^{n} x_{i}^{j_{1} \cdots j_{d}} y_{i}=\sum_{i=1}^{n} x_{i} \otimes y_{i} .
\end{aligned}
$$

This establishes $\ell_{q}\left(\mathbf{N}^{d}\right) \otimes_{q} \ell_{q}(\mathbf{N}) \cong \ell_{q}\left(\mathbf{N}^{d}, \ell_{q}(\mathbf{N})\right)$. 


\section{Approximation in $L_{2}$}

We are now ready to describe the approximation spaces $A_{q}^{\alpha}$ for best $N$ term approximation in $L_{2}\left([0,1]^{d}\right)$ using the anisotropic ("sparse grid") tensor product basis $\left\{\psi_{j_{1} k_{1} \cdots j_{d} k_{d}}\right\}$.

Definition 4. For $d \geq 2$ and $q^{-1}=\alpha+\frac{1}{2}$, we define

$$
\hat{B}_{q}^{\alpha}\left([0,1]^{d}\right):=B_{q}^{\alpha}\left(L_{q}([0,1])\right) \otimes_{q} \cdots \otimes_{q} B_{q}^{\alpha}\left(L_{q}([0,1])\right) .
$$

Using Theorem 4 (resp., Theorem 3 for the case $q \geq 1$ ) and the norm equivalences (4), we get

Lemma 2. For $\alpha<\alpha_{0}$ and $q^{-1}=\alpha+\frac{1}{2}$, we have

$$
\left\|\sum_{j_{i}, k_{i}} c_{j_{1} k_{1}, \ldots, j_{d}, k_{d}} \psi_{j_{1} k_{1}, \ldots, j_{d} k_{d}}\right\|_{\hat{B}_{q}^{\alpha}\left([0,1]^{d}\right)} \sim\left\|\left(c_{j_{1} k_{1}, \ldots, j_{d} k_{d}}\right)\right\|_{\ell_{q}\left(\mathbf{N}^{d}\right)} .
$$

We now prove the Bernstein and Jackson inequalities for the spaces $\hat{B}_{q}^{\alpha}\left([0,1]^{d}\right)$.

Lemma 3 (The Bernstein Inequality). For all $S \in \Sigma_{N}$, we have

$$
\|S\|_{\hat{B}_{q}^{\alpha}\left([0,1]^{d}\right)} \lesssim N^{\alpha}\|S\|_{L_{2}\left([0,1]^{d}\right)} .
$$

Proof. Let $S=\sum_{i=1}^{N} c_{i} \psi_{i}$, where $\psi_{i} \in\left\{\psi_{j_{1} k_{1}} \otimes \cdots \otimes \psi_{j_{d} k_{d}}\right\}$. Using Lemma 2, (3), and the mutual equivalence between finitely supported $\ell_{q}$-norms,

$$
\|x\|_{\ell_{p}(N)} \leq\|x\|_{\ell_{q}(N)} \leq N^{1 / q-1 / p}\|x\|_{\ell_{p}(N)}, \quad x \in \mathbf{R}^{N}, \quad 0<q \leq p \leq \infty,
$$

we get

$$
\|S\|_{\hat{B}_{q}^{\alpha}\left([0,1]^{d}\right)} \sim\left\|\left(c_{i}\right)\right\|_{\ell_{q}(N)} \lesssim N^{1 / q-1 / 2}\left\|\left(c_{i}\right)\right\|_{\ell_{2}(\mathbf{N})} \lesssim N^{\alpha}\|S\|_{L_{2}\left([0,1]^{d}\right)} .
$$

Lemma 4 (The Jackson Inequality). For all $f \in \hat{B}_{q}^{\alpha}\left([0,1]^{d}\right)$, we have

$$
\inf _{S \in \Sigma_{N}}\|f-S\|_{L_{2}([0,1])} \lesssim N^{-\alpha}\|f\|_{\hat{B}_{q}^{\alpha}\left([0,1]^{d}\right)} .
$$

Proof. Since $f \in \hat{B}_{q}^{\alpha}\left([0,1]^{d}\right)$, we have $c \in \ell_{q}\left(\mathbf{N}^{d}\right)$ for the coefficient vector of the wavelet decomposition. Using (3) and Theorem 2, we infer, with $\tau=q$ and $\ell_{q, q}=\ell_{q}$,

$$
\inf _{S \in \Sigma_{N}}\|f-S\|_{L_{2}([0,1])} \lesssim N^{-\alpha}\|c\|_{\ell_{q}\left(\mathbf{N}^{d}\right)} \lesssim N^{-\alpha}\|f\|_{\hat{B}_{q}^{\alpha}\left([0,1]^{d}\right)} .
$$

Eventually, we can apply Theorem 1 to characterize the approximation spaces. 
Theorem 5. The approximation space $A_{q}^{\alpha}\left(L_{2}\left([0,1]^{d}\right)\right), q^{-1}=\alpha+\frac{1}{2}, 0<\alpha<\alpha_{0}$, corresponding to an approximation rate $\alpha$ in $L_{2}\left([0,1]^{d}\right)$ by best $N$ term approximation using the anisotropic tensor product wavelet basis $\left\{\psi_{j_{1} k_{1} \ldots j_{d} k_{d}}\right\}$, is given by

$$
A_{q}^{\alpha}\left(L_{2}\left([0,1]^{d}\right)\right)=\hat{B}_{q}^{\alpha}\left([0,1]^{d}\right) .
$$

Proof. Theorem 1 together with Lemmata 3 and 4 yields

$$
A_{q}^{\alpha}\left(L_{2}\left([0,1]^{d}\right)\right)=\left(L_{2}\left([0,1]^{d}\right), \hat{B}_{s}^{\beta}\left([0,1]^{d}\right)\right)_{\alpha / \beta, q}, \quad s^{-1}=\beta+\frac{1}{2},
$$

for $\beta$ with $\alpha<\beta<\alpha_{0}$. From the isomorphism to $\ell_{q}$-spaces and the corresponding interpolation result,

$$
\left(\ell_{2}, \ell_{s}\right)_{\theta, q}=\ell_{q}
$$

for

$$
\theta=\frac{1 / q-\frac{1}{2}}{1 / s-\frac{1}{2}}=\frac{\alpha}{\beta}
$$

it follows that

$$
\left(L_{2}\left([0,1]^{d}\right), \hat{B}_{s}^{\beta}\left([0,1]^{d}\right)\right)_{\alpha / \beta, q}=\hat{B}_{q}^{\alpha}\left([0,1]^{d}\right) .
$$

\section{Approximation in $H^{1}$}

In this section we treat best $N$ term approximation with respect to the Sobolev $H^{1}\left([0,1]^{d}\right)$-norm. The methods are similiar to the $L_{2}$-case. Therefore, we will not go into much detail here.

Note that we can decompose the space $H^{1}\left([0,1]^{2}\right)$ into an intersection of tensor products of one-dimensional spaces,

$$
H^{1}\left([0,1]^{2}\right) \cong\left(H^{1}([0,1]) \otimes_{2} L_{2}([0,1])\right) \cap\left(L_{2}([0,1]) \otimes_{2} H^{1}([0,1])\right),
$$

and generally (denoting $L_{2}([0,1])$ by $H^{0}([0,1])$ ),

$$
H^{1}\left([0,1]^{d}\right) \cong \bigcap_{k=1}^{d}\left(\bigotimes_{i=1}^{d} H^{\delta_{i k}}([0,1])\right) .
$$

Hence it suffices to treat the case $H^{1}([0,1]) \otimes_{2} L_{2}([0,1]) \otimes_{2} \cdots \otimes_{2} L_{2}([0,1])$.

Rescaling the wavelet basis $\psi_{j k}$ in the first variable, by multiplying with a factor $2^{-j}$, we get a basis normalized in $H^{1}([0,1])$ satisfying the norm equivalence

$$
\left\|\sum_{j, k} c_{j k}\left(2^{-j} \psi_{j k}\right)\right\|_{H^{1}([0,1])} \sim\left\|\left(c_{j k}\right)\right\|_{\ell_{2}(\mathbf{N})},
$$

which will be the substitute for (3); see, e.g., [12], [13]. 
Analogously, the Besov norm equivalences (4) read as, for $1 \leq \alpha<\alpha_{0}$,

$$
\left\|\sum_{j, k} c_{j k}\left(2^{-j} \psi_{j k}\right)\right\|_{B_{q}^{\alpha}\left(L_{q}([0,1])\right)} \sim\left\|\left(c_{j k}\right)\right\|_{\ell_{q}(\mathbf{N})}, \quad q^{-1}=\alpha-\frac{1}{2} .
$$

The spaces corresponding to the remaining variables remain unchanged. Hence, we have norm equivalences

$$
\left\|\sum_{j_{i}, k_{i}} c_{j_{1} k_{1} \ldots j_{d} k_{d}} \psi_{j_{1} k_{1} \ldots j_{d} k_{d}}\right\|_{H^{1}([0,1]) \otimes_{2} L_{2}([0,1]) \otimes_{2} \ldots \otimes_{2} L_{2}([0,1])} \sim\|(c)\|_{\ell_{2}\left(\mathbf{N}^{d}\right)}
$$

as well as

$$
\left\|\sum_{j_{i}, k_{i}} c_{j_{1} k_{1} \ldots j_{d} k_{d}} \psi_{j_{1} k_{1} \ldots j_{d} k_{d}}\right\|_{X} \sim\|(c)\|_{\ell_{q}\left(\mathbf{N}^{d}\right)}
$$

for the space

$$
X=B_{q}^{1 / q+1 / 2}\left(L_{q}([0,1])\right) \otimes_{q} B_{q}^{1 / q-1 / 2}\left(L_{q}([0,1])\right) \otimes_{q} \cdots \otimes_{q} B_{q}^{1 / q-1 / 2}\left(L_{q}([0,1])\right) .
$$

Now the Jackson and Bernstein inequalities imply a characterization of the approximation spaces by interpolation spaces.

Theorem 6. The approximation space $A_{q}^{\alpha}\left(H^{1}\left([0,1]^{d}\right)\right), q^{-1}=\alpha+\frac{1}{2}, 0<\alpha<\alpha_{0}-1$, corresponding to an approximation rate $\alpha$ in $H^{1}\left([0,1]^{d}\right)$ by best $N$ term approximation using the anisotropic tensor product wavelet basis $\left\{\psi_{j_{1} k_{1} \ldots j_{d} k_{d}}\right\}$, is given by

$$
A_{q}^{\alpha}\left(H^{1}\left([0,1]^{d}\right)\right)=\tilde{B}_{q}^{\alpha}\left([0,1]^{d}\right)
$$

where the space $\tilde{B}_{q}^{\alpha}\left([0,1]^{d}\right)$ is defined as

$$
\tilde{B}_{q}^{\alpha}\left([0,1]^{d}\right)=\bigcap_{k=1}^{d}\left(\bigotimes_{i=1}^{d} X_{\delta_{i k}}([0,1])\right)
$$

(q-tensor product) with

$$
X_{0}([0,1])=B_{q}^{\alpha}\left(L_{q}([0,1])\right)
$$

and

$$
X_{1}([0,1])=B_{q}^{\alpha+1}\left(L_{q}([0,1])\right)
$$

Proof. As before, Theorem 1 yields

$$
A_{q}^{\alpha}\left(H^{1}\left([0,1]^{d}\right)\right)=\left(H^{1}\left([0,1]^{d}\right), \tilde{B}_{s}^{\beta}\left([0,1]^{d}\right)\right)_{\alpha / \beta, q}, \quad s^{-1}=\beta+\frac{1}{2},
$$

for $\beta$ with $\alpha<\beta<\alpha_{0}-1$. From the isomorphism to $\ell_{q}$-spaces and the corresponding interpolation result, it follows that

$$
\left(H^{1}\left([0,1]^{d}\right), \tilde{B}_{s}^{\beta}\left([0,1]^{d}\right)\right)_{\alpha / \beta, q}=\tilde{B}_{q}^{\alpha}\left([0,1]^{d}\right) .
$$


Remark 7. In [20] we have proven that solutions to smooth, linear, uniformly elliptic PDEs on polygonal/polyhedral domains with smooth boundary data of Dirichlet, Neumann, or mixed type in dimensions 2 and 3 , as functions transported to the unit cube allow for approximation using tensor products of one-dimensional spline wavelets of local polynomial degree $p \geq 1$ at any rate up to (but not including) $p$ with respect to the $H^{1}$-norm and at any rate up to (but not including) $p+1$ with respect to the $L_{2}$-norm. This is even true for elliptic transmission problems (piecewise smooth coefficients of the elliptic operator with discontinuities along smooth curves). This immediately implies that these functions, as functions transported to the unit cube, belong to the "anisotropic" Besov scales $\hat{B}_{q}^{\alpha}\left([0,1]^{2}\right)$ and $\tilde{B}_{q}^{\alpha}\left([0,1]^{d}\right)$ for all $\alpha>0$.

Remark 8. It is interesting to compare the "tensor product"-Besov regularity of elliptic singularities in two and three dimensions with results involving the classical (isotropic) Besov scales $B_{q}^{\alpha}\left(L_{q}(\Omega)\right), q^{-1}=\alpha / d+\frac{1}{2}$, for polygonal/polyhedral domains $\Omega \subset$ $\mathbf{R}^{d}$. Our results hold for any type of elliptic singularity, even those including elliptic transmission problems, in the entire scales $\hat{B}_{q}^{\alpha}\left([0,1]^{2}\right)$ and $\tilde{B}_{q}^{\alpha}\left([0,1]^{d}\right)$ in dimensions two and three; see Remark 7 above.

Regarding isotropic Besov spaces in dimension 2, we have similiarly elliptic singularities in dimension 2 belonging to the whole scale of Besov spaces $B_{q}^{\alpha}\left(L_{q}(\Omega)\right.$ ), $q^{-1}=\alpha / 2+\frac{1}{2}, 0<\alpha<\infty$, if the data of the problem are smooth but the domain polygonal, see [10]. This fits perfectly well with the fact that adaptive methods allow us to recover the optimal isotropic rate (which is, of course, only $p / 2$ in $H^{1}$ compared to $p-\varepsilon$ for arbitrarily small $\varepsilon>0$ in appropriate adaptive approximation using tensor product bases) implied by the polynomial degree $p$ of the ansatz functions for isotropically based finite element methods in dimension 2.

In dimension 3 elliptic singularities are known to exhibit anisotropic components along edges. An adaptive method based on isotropically supported basis functions cannot, in general, recover the full isotropic rate (which would be $p / 3$ in $H^{1}$ ), see, e.g., [2]. And, correspondingly, it has not been proven yet that elliptic singularities in dimension 3 belong to the whole scale of isotropic Besov spaces $B_{q}^{\alpha}\left(L_{q}(\Omega)\right), q^{-1}=\alpha / 3+\frac{1}{2}$; only a result with strong restrictions on $\alpha$ (about $\leq 3$ ) is known, see [11].

It therefore seems that our notion of anisotropic Besov regularity is more appropriate to describe the regularity of solutions to elliptic problems than regularity based on isotropic Besov spaces.

Our regularity results are not confined to the geometry of the unit cube but apply to any type of elliptic singularity transported as a function to the unit cube.

Remark 9. The examples of approximation in $L_{2}\left([0,1]^{d}\right)$ and $H^{1}\left([0,1]^{d}\right)$ have been chosen since they are of interest to the numerical analyst. Clearly, any appropriate scale of spaces admitting isomorphisms to $\ell_{q}$-spaces gives rise to corresponding approximation results.

Remark 10. In [6] and in papers [7], [8], and [3], a numerical algorithm has been developed which asymptotically realizes best $N$ term approximation rates for solutions to elliptic equations under mild assumptions on the elliptic operator. In the author's 
thesis [21], we apply this algorithm to the tensor product setting. We show that if the data are given in tensor product structure, then the algorithm [6] applies and realizes asymptotically the substantially higher "sparse grid" rates for elliptic problems.

Acknowledgments. The author is a member of the IHP network "Breaking Complexity" of the EC, contract No. HPRN-CT-2002-00286, and was supported by the Swiss Federal Office for Science and Education under grant BBW02.0418. The author would also like to thank his thesis adviser Christoph Schwab for many helpful discussions, and also Radu Alexandru Todor for contributing to Lemma 1.

\section{References}

1. ST. ACHATZ (to appear): Higher order sparse grid methods for elliptic partial differential equations with variable coefficients. Special Issue of Computing on Sparse Grids.

2. TH. Apel (1999): Anisotropic Finite Elements: Local Estimates and Applications. Advances in Numerical Mathematics. Stuttgart: Teubner.

3. A. Barinka, T. Barsch, P. Charton, A. Cohen, S. Dahlke, W. Dahmen, K. Urban (2001): Adaptive wavelet schemes for elliptic problems-Implementations and numerical experiments. SIAM J. Sci. Comput., 23(3):910-939.

4. C. BenNetT, R. Sharpley (1988): Interpolation of Operators. New York: Academic Press.

5. P. BINEV, W. DAHMEN, R. A. DEVORE (2004): Adaptive finite element methods with convergence rates. Numer. Math., 97(2):219-268.

6. A. COHEN, W. DAHMEN, R. A. DEVORE (2000): Adaptive wavelet methods for elliptic operator equations: Convergence rates. Math. Comp., 70(233):27-75.

7. A. Cohen, W. Dahmen, R. A. DeVore (2002): Adaptive wavelet methods II-Beyond the elliptic case. Found. Comput. Math., 2(3):203-245.

8. A. COHEN, W. DAHMEN, R. A. DeVore (2003): Adaptive wavelet schemes for nonlinear variational problems. SIAM J. Numer. Anal., 41(5):1785-1823.

9. A. COHEN, R. A. DeVore, R. Hochmuth (2000): Restricted nonlinear approximation. Constr. Approx., 16:85-113.

10. S. DAHLKE (1999): Besov regularity for elliptic boundary value problems in polygonal domains. Appl. Math. Lett., 12:31-36.

11. S. DAHLKE (2002): Besov regularity of edge singularities for the Poisson equation in polyhedral domains. Numer. Linear Algebra Appl., 9:457-466.

12. W. DAhmen, A. KunOth, K. URBAn (1999): Biorthogonal spline-wavelets on the interval-Stability and moment conditions. Appl. Comput. Harmon. Anal., 6:132-196.

13. W. DAHMEN, R. SCHNEIDER (1998): Wavelets with complementary boundary conditions-Function spaces on the cube. Result. Math., 34:255-293.

14. R. A. DeVore, G. G. LORENTZ (1993): Constructive Approximation. Grundlehren der Mathematischen Wissenschaften. Berlin: Springer-Verlag.

15. R. A. DeVore (1998): Nonlinear Approximation. Acta Numerica, Vol. 7. Cambridge, UK: Cambridge University Press, pp. 51-150.

16. M. GRIEBEL, S. KNAPEK (2000): Optimized tensor-product approximation spaces. Constr. Approx., 16(4):525-540.

17. N. KALTON (2003): Quasi-Banach spaces. In: Handbook of the Geometry of Banach Spaces, Vol. 2, pp. 1099-1130. Amsterdam: North-Holland.

18. W. Light, E. Cheney (1980): Approximation Theory in Tensor Product Spaces. Lecture Notes in Mathematics, Vol. 1169. New York: Springer-Verlag.

19. P. MORIN, R. NOCHETTO, K. SIEBERT (2000): Data oscillation and convergence of adaptive FEM. SIAM J. Numer. Anal., 38(2).

20. A. NITSCHE (2001): Sparse approximation of singularity functions. Constr. Approx., 21(1):63-81. 
21. A. Nitsche (2004): Sparse Tensor Product Approximation of Elliptic Problems. Dissertation an der Eidgenössischen Technischen Hochschule ETH Zürich, Switzerland.

22. P. OsWALD (1999): On best n-term approximation by Haar functions in $H^{s}$-norms. In: Metric Function Theory and Related Topics in Analysis (Nicolskij, Kashin, and Izaak, eds.). Moscow: AFC, pp. 137-163.

23. P. OSWALD (1999): Best $n$-term capacitance approximation on sparse grids. In: Proc. 12th Intern. Conf. on Domain Decomp. Methods in Science and Engineering (T. Chan et al., eds.). Chiba, pp. 437-444.

24. J. PEETRE (1968): A Theory of Interpolation of Normed Spaces. Course Notes. University of Brasilia.

25. S. A. SMOLYAK (1963): Quadrature and interpolation formulas for tensor products of certain classes of functions. Soviet Math. Dokl., 4:240-243.

26. V. TEMLYAKOV (2002): Nonlinear methods of approximation. Found. Comput. Math., 3:33-107.

27. P. TURPIN (1982): Représentation fonctionelle des espaces vectorielles topologiques. Studia Math., 73:1-10.

28. CH. ZENGER (1991): Sparse grids; in parallel algorithms for PDE. In: Proc. 6th GAMM Seminar, Kiel (W. Hackbusch, ed.). Braunschweig: Vieweg, pp. 241-251.

P.-A. Nitsche

Seminar for Applied Mathematics

ETH-Zentrum

CH-8092 Zurich

Switzerland 